\title{
AN IMPROVED METHOD FOR RETAINING POLYANILINE ON GLASS SURFACE
}

\author{
R.M.G. RAJAPAKSE* and D.K.W.W.M.S. WICKRAMANAYAKE \\ Department of Chemistry, University of Peradeniya, Peradeniya.
}

(Received: 24 March 1999 ; accepted: 05 November 1999)

\begin{abstract}
Abstrct: An improved method for obtaining polyaniline retained glass plates having considerably higher electrical conductivity is described. In this method, precleaned glass plates were refluxed with conc. $\mathrm{HCl}$ and then rinsed with distilled water: The plates were heated at $400^{\circ} \mathrm{C}$, and the surface hydroxyl groups were replaced with $\mathrm{NHC}_{6} \mathrm{H}_{5}$ groups. The deposition of polyaniline on such modified surfaces results in a polyaniline layer chemical bonded to glass plates. The maximum resistance of $1 \mathrm{~cm}$ distance on the conducting surface was $9 \mathrm{k} \Omega$ that is an order of magnitude greater than that prepared without the refluxing step. Only the use of oxidants, $\mathrm{S}_{2} \mathrm{O}_{4}{ }^{2-}(\mathrm{aq})$ and $\mathrm{Fe}^{3+}(\mathrm{aq})$ gave polyaniline layers in their conducting state. The molar ratio of the monomer to oxidant that yields the highest conductivity is $1: 2$. The oxidants such as $\mathrm{Cr}_{2} \mathrm{O}_{7}{ }^{2-}(\mathrm{aq})$ and $\mathrm{MnO}_{4}{ }^{-}$(aq) gave polyaniline layers in their pernigraniline form the same molar ratios. Lowering the $\mathrm{pH}$ of the polymerisation medium produced increased conductivity in the polyaniline coated glass plates. The polyaniline layer hydrolyses when treated with concentrated alkali.
\end{abstract}

Keywords: Electrical conductivity, functionalised glass, polyaniline.

\section{INTRODUCTION}

Polyaniline can exist in six different structural forms depending upon the extent of oxidation and protonation of the polymer backbone. The fully reduced form of the polymer, which can exist only in a neutral or basic medium, is leucoemeraldine(a). This contains only phenyl groups and -NH- linkages. In a sufficiently acidic medium, some of the amine units of leuco-emeraldine can be protonated resulting in a quaternary ammonium salt type of structure. The latter is known as leuco-emeraldine salt(b). These two forms of the polymer are transparent yellow in colour. The partial oxidation of leuco-emeraldine salt results in a green polymer known as emeraldine salt(c). In this form, one out of four phenyl rings have converted to quinoid form with $=\mathrm{N}$ - attached to it on both sides. Due to partial oxidation, the polymer contains some cation radicals known as polarons. Neutralisation of the emeraldine salt gives emeraldine base(d), which is blue in colour. Futher oxidation of the emeraldine salt gives a violet polymer called pernigraniline salt(e). Neutralisation of pernigraniline salt gives pernigraniline base $(f)^{1}$. While the first four forms of the polymer are remarkably stable in air and in solution, the fully oxidised form is prone to various 
chemical reactions and thereby degradation ${ }^{2}$. Only the emeraldine salt form of the polymer is an electrical conductor with maximum recorded conductivity of $100 \mathrm{~S}$ $\mathrm{cm}^{-1}$ while all other forms are electrical insulators. Forms (a) and (c) can be interconverted by chemical redox agents or electrochemically by switching applied potential between $-0.2 \mathrm{~V}$ to $+0.5 \mathrm{~V}$ with respect to the saturated calomel electrode (wrt SCE). This process is known as redox switching. The redox switching can be achieved in a millisecond time scale with concomitant colour change (from yellow to green or vice versa). Hence the application of the material in electrochromic devices has already been suggested by many authors. In fact, a recent report shows that the device has become a reality ${ }^{3}$.

The forms (c) and (d) or (a) and (b) may be interconverted by the acid/base treatment. The concomitant colour change has been utilised in devising sensors for non-redox basic gases such as $\mathrm{NH}^{4}$ and for non-redox acidic gases such as $\mathrm{HCl}$ in the form of fumes ${ }^{4}$. Further the conductivity and colour change associated with redox switching of polyaniline have been utilised in fabricating sensors for both gaseous and solution phase redox agents ${ }^{4}$. The counter ion or proton influx or egress associated with redox switching has been utilised in devising sensors for anions and for $\mathrm{pH}^{5,6}$. Polyaniline was found to be a good electrocatalyst for L-ascorbic acid oxidation ${ }^{7}$. Precious metal doped polyaniline has been used in electrocatalytic oxidation of methanol, ethylene glycol, glucose etc. ${ }^{s}$. Polyaniline based various electronic and electrical devices including rechargeable batteries have already been fabricated ${ }^{9,10}$.

Most of the above technological applications of the polymer undoubtedly demand polyaniline coated glass plates. The usual procedure for this is solution casting. One of the problems with polyaniline and such other conducting polymers is their extreme insolubility in common solvents. However, recent advances in conducting polymer research enabled the development of various processing techniques for them. For instance, when polyaniline is doped with campour sulphonic acid or such other surfactants, the resulting materials are soluble in N-methyl pyrrolidinone ${ }^{11}$. By modifying the polymer to possess pendant or interacting polar groups, water soluble-polyanilines were also developed ${ }^{12}$. The polymer films obtained on glass surfaces by solvent casting are, however, not firmly attached to glass surfaces. These films peel off on abrasion.

Recently, we reported a method to retain polyaniline onto ordinary glass surfaces functionalised with anilin $\mathrm{e}^{13}$. In this procedure, pre-cleaned ordinary glass pieces were pre-heated at $400^{\circ} \mathrm{C}$ and the surface $-\mathrm{OH}$ groups were replaced by $-\mathrm{Cl}$ groups by $\mathrm{SOCl}_{2}$ treatment at 400 "C. The $\mathrm{Si}-\mathrm{Cl}$ groups were then converted. to $\mathrm{Si}-\mathrm{NHC}_{6} \mathrm{H}_{5}$ groups. This has resulted in aniline functionalised glass. The polymerisation of aniline on such surfaces by $\mathrm{S}_{2} \mathrm{O}_{8}{ }^{2-}$ in an acidic aqueous solution generated polyaniline grafted onto glass surfaces. The lowest resistance of $1 \mathrm{~cm}$ length of the polymer coat was $306 \mathrm{k} \Omega$. The material was found to be a good template 
for aligning liquid crystalline molecules in liquid crystal display devices ${ }^{14}$. The material has also shown to be attractive in fabricating gas sensors. However, the conductivity of the material is insufficient for use as an electrochromic device with a fast switching time. The application of the material as electrodes in rechargeable batteries is not possible due to its low conductivity. These obstacles led us to devise an improved protocol to graft polyaniline onto glass plates to give a higher electrical conductivity.

\section{METHODS AND MATERIALS}

All chemicals used were of AnalaR grade and were purchased from Aldrich Chemical Company, UK. The aniline used was distilled under nitrogen and other chemicals were used without further purification. Glass plates used were high-quality pre-cleaned plates.

In the present work, glass plates $(1 \mathrm{~cm} \times 1 \mathrm{~cm})$ were refluxed in concentrated $\mathrm{HCl}$ for varying lengths of times $(2,4,6,7,8,10,24 \mathrm{~h})$. The treated glass plates were rinsed well with distilled water, and allowed to dry. They were then heated on a pre-heated hot plate at ca. 400 " $\mathrm{C}$ for $30 \mathrm{~min}$ in a fume cupboard. $\mathrm{SOCl}_{2}$ was then sprayed onto their exposed surfaces under the above conditions, for $2 \mathrm{~min}$. This spray was then followed by a spray of dry, distilled aniline for further two min. In certain experiments, the spraying of $\mathrm{SOCl}_{2}$ and aniline was repeated twice. The treated glass plates were then rinsed with distilled water several times and placed in a solution containing aniline, $\mathrm{S}_{2} \mathrm{O}_{8}{ }^{2} \cdot(\mathrm{aq})$ and an acid $\left(\mathrm{HCl}, \mathrm{H}_{2} \mathrm{SO}_{4}, \mathrm{H}_{3} \mathrm{PO}_{4}, \mathrm{HNO}_{3}\right.$ or $\mathrm{HClO}_{4}$ ) whose concentrations were selected as shown in Table 2 . In order to investigate the best oxidant that gives polyaniline coated glass plates with highest conductivity, oxidants such as acidic aqueous solutions of $\mathrm{Fe}^{3+}, \mathrm{MnO}_{4}^{-}, \mathrm{Cr}_{2} \mathrm{O}_{7}{ }^{2-}$ or $\mathrm{H}_{2} \mathrm{O}_{2}$ were used in place of $\mathrm{S}_{2} \mathrm{O}_{8}{ }^{2-}$ (Table 3 ). The effect of $\mathrm{pH}$ of the polymerisation solution on the conductivity of the polymer coat obtained was also studied by varying the $\mathrm{HCl}$ concentration but keeping all other variables constant (Table 4). The polymer bound glass plates were air-dried and stored in a dry desiccater. The resistance of a $1 \mathrm{~cm}$ length of the polymer coat on the glass plate was measured using a high impedance multimeter at different locations of the polymer coat and found to be almost constant. The polymer coats were uniform when observed through an optical microscope.

In order to investigate the side of attachment of aniline (i.e., via $\mathrm{Si}-\mathrm{N}$ or via Si-C linkages) on the glass surface, the ordinary pre-cleaned glass pieces, aniline attached glass pieces and polymer attached glass pieces were placed in a diazotisation mixture at $0-5$ " $\mathrm{C}$ for several hours. The glass plates were then removed from the mixture, rinsed well with doubly distilled water and placed in a solution containing $\beta$-naphthol and $\mathrm{NaOH}$. The colour changes and other effects of the above chemical treatments were investigated. 


\section{RESULTS}

Table 1 shows the dependence of the surface conductivity of the polyaniline bound glass surfaces on the length of refluxing the glasses with conc. HCl prior to polyaniline attachment. In all these experiments, the composition of the polymerisation solution was fixed at $1 \mathrm{~mol} \mathrm{dm}-3 \mathrm{HCl}, 0.1 \mathrm{~mol} \mathrm{dm}^{-3}$ aniline and $0.2 \mathrm{~mol} \mathrm{dm}^{-3} \mathrm{~S}_{2} \mathrm{O}_{8}^{2-}$. As can be seen from this Table, the conductivity of the polymer coat increases as the refluxing time is increased from $2 \mathrm{~h}$ to $6 \mathrm{~h}$ and then it remains almost unchanged.

Table 1: The dependence of the surface conductivity of the polyaniline attached glass plates with the length of refluxing with conc. HCl. The composition of the polymerisation mixture is $1 \mathrm{~mol} \mathrm{dm}^{-3} \mathrm{HCl}, 0.1 \mathrm{~mol} \mathrm{dm}^{-3}$ aniline and $0.2 \mathrm{~mol} \mathrm{dm}{ }^{-3} \mathrm{SO}_{2} \mathrm{O}_{8}^{2 .}$

The conductivity of the polyaniline coated surface depends on the nature of the acid used to adjust the $\mathrm{pH}$ of the polymerisation solution and also on the monomer to oxidant concentration ratio. These variations are shown in Table 2. Results show that the nature of the acid used has an effect on the conductivity of the polymer coat. Among the acids chosen use of $\mathrm{HNO}_{3}$ or $\mathrm{HCl}$ gives the polymer coats with lowest resistance per $1 \mathrm{~cm}$ length at $1: 2$ monomer: $\mathrm{S}_{2} \mathrm{O}_{8}{ }^{2-}$ concentration ratio. Also the use of oxidants such as $\mathrm{MnO}_{4} ; \mathrm{Cr}_{2} \mathrm{O}_{7}^{2-}$ or $\mathrm{H}_{2} \mathrm{O}_{2}$ always resulted in non-conducting polymer coats while $\mathrm{Fe}^{3+}$ and $\mathrm{S}_{2} \mathrm{O}_{8}{ }^{2-}$ gave conducting polymer coats (Table 3 ). $\mathrm{Fe}^{3+}$ seems to be a better oxidant than $\mathrm{S}_{2} \mathrm{O}_{8}{ }^{2}$ in this respect. Thus when $\mathrm{Fe}^{3+}$ was used as the oxidant at the optimum monomer to oxidant ratio of $1: 2$, the decrease in $\mathrm{pH}$ of the polymerisation solution has resulted in increased conductivity for the polymer coat obtained (Table 4). The monomer bound and polymer bound glass plates were exposed to various acids, bases and redox agents and their effects are depicted in Table 5. 
In the $\beta$-naphthol coupling experiment to diasotized amine groups, the characteristic pink colouration was immediately observed with polymer modified glass plates. The polymer layer that had turned pink was peeled off from the glass surface upon ageing. There was no immediate colour change in the aniline modified glass plates. However, pink colouration was observed in this case also with ageing and the coating on the glass then peeled off. There was no pink colouration when ordinary pre-cleaned glass plates were subjected to the above chemical treatments.

Table 2: The dependence of the conductivity of polyaniline grafted glass plates when different acids are used to adjust $\mathrm{pH}$ of the polymerisation solution. The concentration of aniline chosen was $0.250 \mathrm{~mol} \mathrm{dm}^{-3}$ in all experiments. The oxidant $\mathrm{S}_{2} \mathrm{O}_{8}^{2-2}$ to monomer concentration ratio was changed from 1:2 to 3:2 in each case.

\begin{tabular}{|c|c|c|}
\hline Acid used & {$\left[\mathrm{S}_{2} \mathrm{O}_{8}^{2-}\right] / \mathrm{mol} \mathrm{dm}^{-3}$} & $\begin{array}{l}\text { Resistance of } 1 \mathrm{~cm} \text { length of the } \\
\text { polymer coat } / \mathrm{k} \Omega\end{array}$ \\
\hline \multirow{3}{*}{$\mathrm{HNO}_{3}$} & 0.125 & 562 \\
\hline & 0.250 & 75 \\
\hline & 0.375 & 70 \\
\hline \multirow[t]{3}{*}{$\mathrm{HCl}$} & 0.125 & 150 \\
\hline & 0.250 & 75 \\
\hline & 0.375 & 65 \\
\hline \multirow[t]{3}{*}{$\mathrm{HClO}_{4}$} & 0.125 & 115 \\
\hline & 0.250 & 110 \\
\hline & 0.375 & 140 \\
\hline \multirow[t]{3}{*}{$\mathrm{H}_{3} \mathrm{PO}_{4}$} & 0.125 & 700 \\
\hline & 0.250 & 275 \\
\hline & 0.375 & Non-conducting \\
\hline \multirow[t]{3}{*}{$\mathrm{H}_{2} \mathrm{SO}_{4}$} & 0.125 & 275 \\
\hline & 0.250 & 175 \\
\hline & 0.375 & 80 \\
\hline
\end{tabular}


Table 3 : The dependence of the conductivity of polyaniline grafted glass plates when different oxidants are used to polymerise aniline. The concentration of aniline chosen was $0.250 \mathrm{~mol} \mathrm{dm}$ in all experiments. The oxidant to monomer concentration was also changed in each case. $\mathrm{HCl}$ was chosen as the acid and its concentration was fixed at $1 \mathrm{~mol} \mathrm{dm}{ }^{-3}$.

\begin{tabular}{|c|c|c|}
\hline Oxidant & {$\left[\right.$ Oxidant] $/ \mathrm{mol} \mathrm{dm}^{-3}$} & $\begin{array}{c}\text { Resistance of } 1 \mathrm{~cm} \text { length of the } \\
\text { polymer coat } / \mathrm{k} \Omega\end{array}$ \\
\hline \multirow{3}{*}{$\mathrm{H}_{2} \mathrm{O}_{2}$} & 0.100 & Non-conducting \\
\hline & 0.250 & Non-conducting \\
\hline & 0.400 & Non-conducting \\
\hline \multirow{3}{*}{$\mathrm{SO}_{2}^{2-}$} & 0.100 & 60 \\
\hline & 0.250 & 16 \\
\hline & 0.400 & 14 \\
\hline \multirow{3}{*}{$\mathrm{Cr}_{2} \mathrm{O}_{7}^{2-}$} & 0.030 & Non-conducting \\
\hline & 0.080 & Non-conducting \\
\hline & 0.130 & Non-conducting \\
\hline \multirow[t]{3}{*}{$\mathrm{Fe}^{3+}$} & 0.200 & 20 \\
\hline & 0.500 & 9 \\
\hline & 0.800 & 11 \\
\hline \multirow{3}{*}{$\mathrm{MnO}_{4}^{-}$} & 0.040 & Non-conducting \\
\hline & 0.100 & Non-conducting \\
\hline & 0.160 & Non-conducting \\
\hline
\end{tabular}

Table 4: The dependence of the conductivity of polyaniline grafted glass plates when $\mathrm{pH}$ of the polymerisation solution was changed. The concentration of aniline chosen was $0.250 \mathrm{~mol} \mathrm{dm}{ }^{-3}$ in all experiments. The oxidant, $\mathrm{Fe}^{3+}$ was chosen at [monomer]: [nxidant $]=1: 2$. HCl was used as the acid.

$\mathrm{pH}$ of the polymerisation solution

Resistance of $1 \mathrm{~cm}$ length of the polymer coat/k $\Omega$ 
Table 5 : The chemical stability of polyaniline grafted glass plates towards various acids, bases and redox agents.

\begin{tabular}{|c|c|}
\hline Reagent used & Inference \\
\hline Dilute acid ( $\mathrm{HCl}, \underset{2}{\mathrm{H}} \mathrm{SO}, \mathrm{H}_{3} \mathrm{PO}$ etc.) & No change \\
\hline Conc. acid ( $\mathrm{HCl}, \mathrm{H}_{2} \mathrm{SO}, \mathrm{H}_{3} \mathrm{PO}$ etc.) & No change \\
\hline $\begin{array}{l}\text { Oxidising agents }\left(\mathrm{S} \mathrm{O}_{2}^{2-}, \mathrm{Fe}^{3+}, \mathrm{Cr}_{2} \mathrm{O}_{7}^{2-}\right. \\
\mathrm{MnO}_{4} \text { etc. }\end{array}$ & $\begin{array}{l}\text { The film did not peel off but colour } \\
\text { changed from green to violet as } \\
\text { expected }\end{array}$ \\
\hline $\begin{array}{l}\text { Reducing agents ( } \mathrm{Sn} / \mathrm{HCl} \text {,hydrazine, } \\
2,4 \text {-dinitrophenylhydrazine, etc.) }\end{array}$ & No change \\
\hline Dilute ammonia & $\begin{array}{l}\text { Colour changed from green to blue } \\
\text { but the film did not peel off. }\end{array}$ \\
\hline Conc. $\mathrm{NaOH}$ & $\begin{array}{l}\text { Colour changed from green to blue } \\
\text { and eventually the polyaniline } \\
\text { layer peeled off }\end{array}$ \\
\hline
\end{tabular}

\section{DISCUSSION}

We have recently shown that aniline-functionalised glass surface retains polyaniline $\mathrm{e}^{11}$. The solubility tests and FT-IR studies of such materials have shown that the polyaniline molecules are most likely to be covalently attached to the glass surface. The attachment should have taken place at positions of the glass plate where surface hydroxyl groups were present. Hence if one could enhance the number of surface hydroxyl groups of glass, one could then attach more polyaniline molecules onto its surface. In a recent study of grafting of silica membrane on glass surfaces, Chentoufi et al have refluxed glass pieces with conc. $\mathrm{HCl}$ for several hours ${ }^{15}$. In addition to hydroxyl groups, the glass surface contains an appreciable amount of Si-O-Si groups. The refluxing then hydrolyses these groups to result in two $\mathrm{Si}-\mathrm{OH}$ groups per each $\mathrm{Si}-\mathrm{O}-\mathrm{Si}$ group. The chemical processes accompanying the refluxing glass with conc. $\mathrm{HCl}$ are shown in Figure 1 . The replacement of $\mathrm{Si}-\mathrm{OH}$ by $\mathrm{Si}-\mathrm{Cl}$ using $\mathrm{SOCl}_{2}$ at an elevated temperature would make the glass surface more reactive than the ordinary one. Aniline then reacts with it to form Si-NHC $\mathrm{H}_{5}$ with the elimination of $\mathrm{HCl}$ (Figure 2). As Table 1 depicts, the refluxing helps to bind more polyaniline molecules onto the glass surface resulting in increased conductivity of the final material. We see again that refluxing for approximately $6 \mathrm{~h}$ is required to hydrolyse a maximum possible number of Si-O-Si groups and beyond that there is not much of a change of number of surface $\mathrm{OH}$ groups. The maximum conductivity of the glass functionalised with polyaniline 
prepared according to this improved procedure is an order of magnitude greater than that without pre-refluxing.
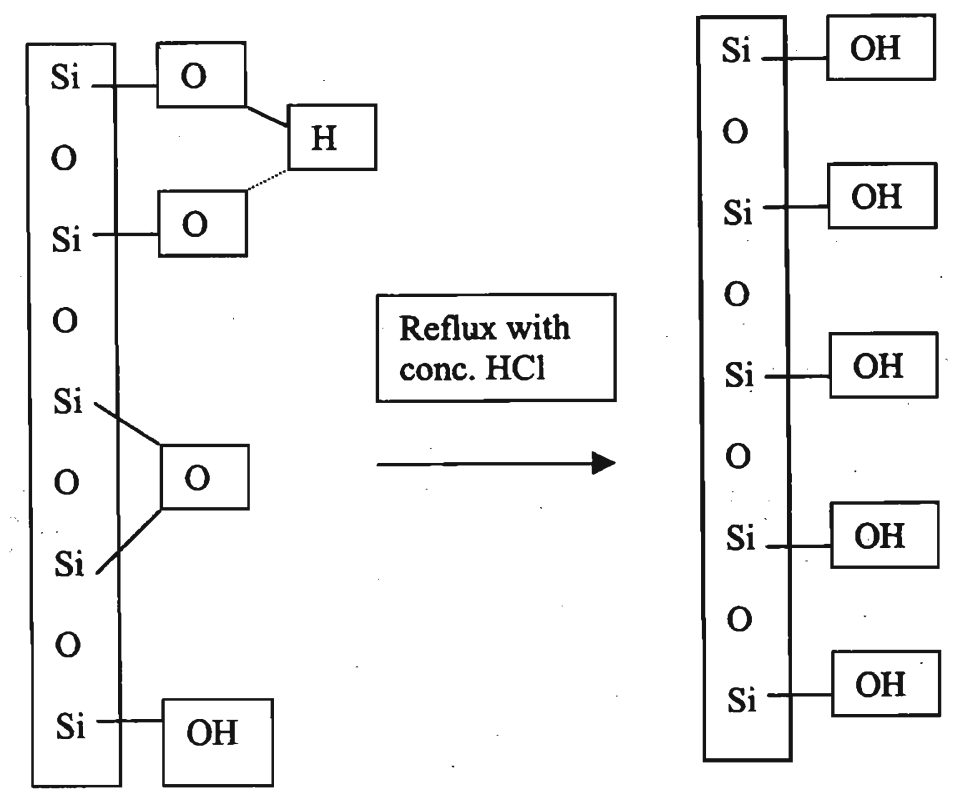

\section{Ordinary glass surface contains surface - OH groups, some of which are hydrogen bonded, and Si-O-Si linkages.}

Glass surface with increased number of surface $-\mathrm{OH}$ groups.

Figure 1 : A method to enhance surface hydroxyl groups of silica glass.

According to the results shown in Table 2, it is clear that whatever the acid used, among the set selected, the surface conductivity of the final product is enhanced when [aniline]: $\left[\mathrm{S}_{2} \mathrm{O}_{8}{ }^{2-}\right.$ ] reaches 1:2. This is in accordance with our previous results ${ }^{14}$. The counter ion introduced by the acid also has a weak effect on the conductivity of the final product. When HCl was used as the acid, the conductivity of the plates were a little higher than those prepared using other acids. It may be explained by considering the necessity of counter ion influx for the emeraldine salt form of the polymer, in order to counterbalance the positive charge of its backbone. The electrochemical quartz crystal microbalance studies have revealed that counter ion influx is occurring during the redox switching of leuco-emeraldine to emeraldine salt form. During this process, unlike other anions, which are trapped in their dehydrated forms, the $\mathrm{Cl}^{-}$ion carries its hydration sphere to some extent ${ }^{16}$. Hence, the polymer always contains some water molecules in it when $\mathrm{Cl}^{-}$ion is used as the counter ion and as a result one could expect higher conductance. 

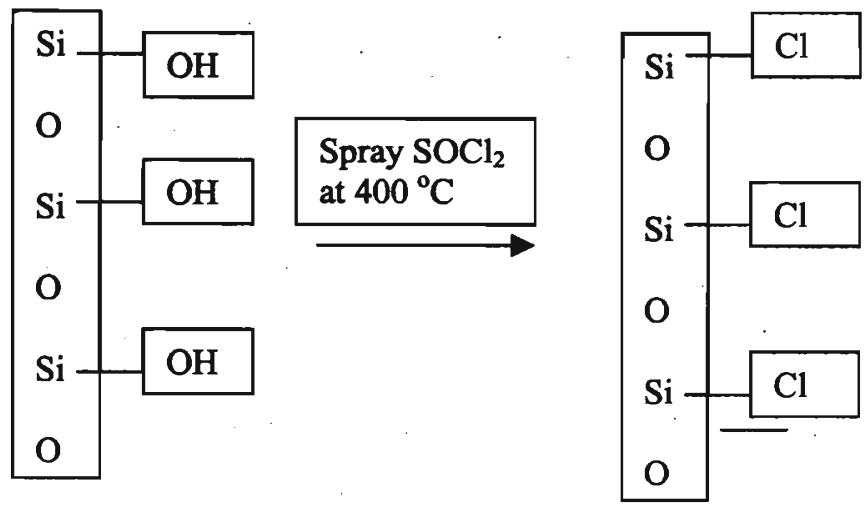

Ordinary glass refluxed with conc. $\mathrm{HCl}$ for at least 6 hours.

\section{Surface}

chlorinated glass

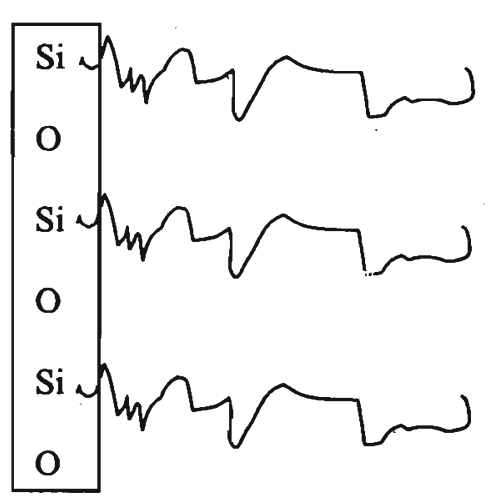

Glass with polyaniline retained on its surface.

The surface conductivity of this glass is an order of magnitude greater than that prepared without refluxing step.

Glass with $\mathrm{NHC}_{6} \mathrm{H}_{5}$ attached onto its surface.

Figure 2: An improved procedure for obtaining polyaniline attached glasses. 
As can be seen from Table 3 , only $\mathrm{S}_{2} \mathrm{O}_{\mathrm{s}}{ }^{2-}$ and $\mathrm{Fe}^{3+}$ ions give conducting glass plates. Although polymer coats were obtained with other oxidants they were in their non-conducting states. With $\mathrm{Cr}_{2} \mathrm{O}_{7}{ }^{2-}$ and $\mathrm{MnO}_{4}-$ the polymer coats obtained were violet in colour. They were in their pernigraniline state despite the lower concentrations of oxidants used by considering the possibility for $6 \mathrm{e}$ and $5 \mathrm{e}$ oxidation by these ions, respectively. Despite the highest redox potential of $\mathrm{S}_{2} \mathrm{O}_{8}{ }^{2-}$, it gives the polymer in its conducting emeraldine salt form. The conductivities of polyaniline coated glass plates, obtained using $\mathrm{S}_{2} \mathrm{O}_{8}{ }^{2-}$ as the oxidant, were comparable to those obtained with $\mathrm{Fe}^{3+}$ as the oxidant. Finally, the $\mathrm{pH}$ of the polymerisation solution has a decisive effect on the polymer coat obtained. The lower the $\mathrm{pH}$ of the polymerisation solution the higher the conductivity of the final product (Table 4).

The stability of Si-N groups will undoubtedly determine the shelf-life of the devices fabricated using these materials. The results in Table 5 clearly show that the polymer coated glass plates can stand for robust conditions and are stable towards strong acids, reducing and oxidising agents. Although the polymer does not peel off when treated by exposing to a weak base the results show that the polymer peels off when treated with a concentrated strong base for a sufficiently long period of time. This can be explained by considering the chemistry of Si-N linkages. The Si-N bond may undergo base hydrolysis under drastic conditions and hence the covalent attachment site of the polymer to the glass surface breaks resulting in the removal of the polymer from the glass surface. This experiment gives an additional support to the retention of polymer on the glass by covalent attachment. The $\beta$-naphthol experiment reveals that there are no free amine groups in aniline attached glass surfaces. Hence, aniline molecules must have attached to the glass surface through Si-N linkages with hanging phenyl groups. With time however, Si-N bond undergoes base hydrolysis releasing free aniline molecules on the glass surface and this explains the pink colouration and peeling off the coat upon ageing. When the polymer is formed on such surfaces, one cannot expect all the polymer molecules to join in the linear chain starting from the monomer unit attached to the glass surface. Although there are more tendencies for the formation of such attached polymer molecules, the presence of polymer chains deposited in random manner is inevitable. This would leave some end $\mathrm{NH}_{2}$ groups in the polymer coated glass plate to give the characteristic pink colouration with $\beta$-naphthol. With ageing, the Si-N bonds undergo base hydrolysis and hence the pink layer peels off from the glass surface. The ordinary pre-cleaned glass surface does not contain any amine group and hence no response to this chemical treatment.

\section{Acknowledgement}

Research support from the National Science Foundation (formerly NARESA) '( $\mathrm{RG} / 98 / \mathrm{C} / 01)$ is gratefully acknowledged. 


\section{References}

1 Skaarup S., Gunaratne L.M.W.K., West K. \& Zachau-Christiansen B. (1995). Polyaniline: influence of polymerisation current density. Materials Research Society Symposium Proceedings 309:565-573.

2 Kobayasi T., Yoneyama H. \& Tamura H. (1984) Journal of Electroanalytical Chemistry 161:419-422.

3 Prakash R. \& Santhanam K.S.V. (1998). Electrochromic window based on polyaniline. Journal of Solid State Electrochemistry 2(2):123-125.

4 Dawan S.K., Kumar D., Ram .K., Chandra S. \& Irivedi D.C. (1997). Application of conducting PANI as sensor material for ammonia. Sensors and Actuators B40:99-103.

5 Li X. \& Li X. (1997). Polyaniline electrode as a detector for electroinactive anions. Gungneng Cailiao 28(3):328-330.

6 Van der Leur R.H.M., Van der Waal A. \& de Hann P.H. (1997). Polyaniline: a conducting polymer for $\mathrm{pH}$ sensors. Proceedings of $5^{\text {th }}$ European Conference on Advanced Materials, Processes and Applications 3:3/463-3/466.

7 Cai L.T. \& Chen H.Y. (1998). Preparation and electroactivity of polyaniline poly(acrylic acid) film electrodes modified by platinum microparticles. Journal. of Applied Electrochemistry 28(2):161-166.

8 Zhou D.M., XuHong J.J., Chen Y. \& Fang Q.H. (1997). Ascorbate sensor based on "self-doped" polyaniline. Electroanalysis 9(15):1185-1188.

9 Chi-Tsuo K., Show An C., Gue-Wu H. \& Hsino-Hung K (1998). Field-effect transistors with the water soluble self-acid-doped polyaniline thin films semiconductor. Synthetic Metals 93(3):155-160.

10 Masau A., Keisuke Y., Yoshihiro U. \& Akira A. (1999). Japanese Patent, Kokai Tokkyo Koho, JP 07 86,093.

11 Cao Y., Smith P. \& Heeger A.J. (1992). Counter ion induced processibility of conducting polyaniline and polyblends of polyaniline in bulk polymers. Synthetic Metals 48(1):91-97.

12 Lee S.B., Lee C.J. \& Wang W.H. (1998). Water-soluble electrically conductive polyaniline composite and its manufacture. Kokai Tokkyo Koho, JP 1092,220, $8 \mathrm{pp}$. 
13 Rajapakse R.M.G., Chandanie A.D.L., Lankeshwara L.P.P. \& Kumarasiri N.L.W.L. (1996). Retention of polyaniline on modified glass surfaces. Synthetic Metals 83:73-76.

14 Chandanie A.D.L., Rajapakse R.M.G., Lankeshwara L.P.P. \& Kumarasiri N.L.W.L. (1997). Novel liquid crystal display on polyaniline modified glass. Molecular Crystals Liquid Crystals 307:125-133.

15 Chentouti M., Dlimi A., Frouji A., Elmidaoui C., Guizard C. \& Cot L. (1998). Seperation des melanges organiques par membranes inorganiques greffees en pervaporation. C.R. Acadamia Scientifica Paris, t.1, Serie II C, 503-508.

16 Lyons M.E.G. (1994). Charge percolation in electroactive polymers. In: Electroactive polymer electrochemistry. (Ed. M.E.G. Lyons) pp 1-226, Plenum Press, USA. 